\title{
A Label-free and Universal Platform for the Construction of Various Logic Circuits Based on Graphene Oxide and G-Quadruplex Structure
}

\author{
Yingying Zhang, ${ }^{*}$ Luhui WANG, ${ }^{* *}$ and Yafei Dong ${ }^{* * * * * *}$ \\ *School of Computer Science, Shaanxi Normal University, Xi'an, 710119, China \\ **College of Life Sciences, Shaanxi Normal University, Xi'an, 710119, China \\ ***Engineering Laboratory for Resource Developing of Endangered Chinese Crude Drugs in Northwest of China, \\ Xi'an, 710119, China
}

\begin{abstract}
Due to structual polymorphism, excellent binding activity and functional significances in biological regulation, G-quadruplex has become the focus of research as an innovated module for analytical chemistry and biomedicine. Meanwhile, in the biosensor fields, new nanomaterial graphene oxide (GO) has also received extensive attention due to its brilliant physical and chemical properties. Herein, we propose a non-label and enzyme-free logic operation platform based on G-quadruplex structure and GO instead of any expensive modification. Taking advantage of the quenching ability of GO to AgNCs and the fluorescence enhancement of NMM ( $N$-methylmesoporphyrin IX) mediated by the split G-quadruplex, a series of binary logic gates (AND, OR, INHIBIT, XOR) have been constructed and verified by biological experiments. Subsequently, two combinatorial logic gates were successfully realized conceptually on the basis of the same BGG platform, including half adder and half subtractor. Taken together, such a universal platform has great potential in applications, such as biocomputing, bio-imaging and disease diagnosis, which cultivate a new view for future biological research.
\end{abstract}

Keywords Molecular logic gates, half adder/half subtractor, graphene oxide, G-quadruplex, label-free

(Received July 26, 2018; Accepted September 25, 2018; Published February 10, 2019)

\section{Introduction}

The rapid development of the electronic computer has played a great role in the molecular field, and molecular science is conversely expected to be an approach available to circumvent any miniaturization limitation of the conventional semiconductor electronic computer. ${ }^{1}$ Especially, molecular computing, data processing at the molecular level, has attracted considerable attention and interest in the area of information technology. ${ }^{2,3}$ Recent years, logic devices based on biochemical or chemical properties have received great development, and further boost the growth trend of complex logic computation with ingeniously designed biomolecules as the basic unit. ${ }^{4,5}$ A variety of bimolecular logic gates that perform basic and advanced logic circuits have been developed, including AND, OR, INHIBIT, ROX, half adder/half subtractor, ${ }^{6}$ full adder/full subtractor, ${ }^{7,8}$ encoder/decoder, ${ }^{9}$ digital comparator ${ }^{10}$ and multiple cascade logic circuit. $^{11}$

In this engineering design and the construction of molecule circuits, DNA is generally accepted to be an attractive building block because of prominent characteristics, such as the parallel processing capability, predictability in design and special recognition of target sequences. ${ }^{12-14}$ In fact, there are many

$\dagger$ To whom correspondence should be addressed.

E-mail: dongyf@snnu.edu.cn kinds of DNA-based logic gates, computational circuits ${ }^{1}$ and automata. ${ }^{15}$ Although some notable progress has been made in constructing biomolecular logic gates, increased computational complexity and high-cost are still major obstacles to the design of advanced logic circuits. The results from the fact that most logic circuits have been implemented on different platforms ${ }^{16}$ and employ modified DNA with fluorophore and quencher. ${ }^{17}$ Given these constraints presented in previous studies, it is essential to design a non-label and universal platform in the field of molecular logic.

Here, instead of any expensive probe modification, G-quadruplex, a special deoxyribonucleotide structure, has been introduced to assist in the model design. In recent years, biosensors based on the G-quadruplex structure have been frequently proposed because of the various reporting signals ${ }^{18-20}$ (colorimetric analysis based on the catalyzing activity of peroxidase-mimicking DNAzymes, ${ }^{21}$ enhanced fluorescence signal with special cationic dyes, ${ }^{22}$ electrochemical ${ }^{23}$ and so on) and multiple design methods (target-triggered formation of G-quadruplex from a blocked state ${ }^{24,25}$ or several split parts ${ }^{26,27}$ ). However, logic devices based on the G-quadruplex are still at a preliminary stage in biological computers and life-science applications. On the other hand, as a kind of advanced nanomaterial, graphene oxide (GO) has attracted great attention in the construction of DNA logic components by utilizing its quenching ability to the fluorescence of various dyes and $\mathrm{AgNCs}$ through long-range fluorescence resonant energy transfer. ${ }^{28}$ 
Table 1 Sequences of oligonucleotides

\begin{tabular}{ll}
\hline Name & \multicolumn{1}{c}{ Sequences $\left(5^{\prime}-3^{\prime}\right)$} \\
\hline BGG & TTGGGTAGGGTCCTTTGTTTGTCGGGTTGGG \\
IN1 & CCCGACAAACAAAGGACCCT \\
IN2 & GGACCCTACCCAATGG \\
IN3 & TTTTTTTTTTGGGTACCGACAAACAAAGGGGTACCCAAAAAAAAAAATCACTA \\
IN4 & ATCGCTAGTGATTTTTTTTTTGGGTACC \\
IN5 & ATCTCATCTCAGTACCCCAACCCGATTGCTGTGTCATAC \\
IN6 & GTATGACACAGCAACCCAACCCGAGTACTGAGATGAGAT \\
IN7 & AGGGTCCTTTGTTTGTCGGG \\
IN8 & ATCTCATCTCAGTACCCCAACCCGATTGCTGTGTCATAC-AgNCs \\
IN9 & GTATGACACAGCAACCCAACCCGAGTACTGAGATGAGAT-AgNCs \\
IN10 & AgNCs-CCCAACCCGACAAACAAAGGATTGGGTAGGG \\
IN11 & GACCCTACCCAATCCTTTGTTTGTCGGTTGTTTTTTTTTTT \\
\hline
\end{tabular}

At the same time, it has been reported that GO can adsorb ss-DNA with high affinity via molecular interactions, but not ds-DNA and G-quadruplex. This feature makes it flexible for modulating the output in biomolecular logic operations. ${ }^{29}$

Inspired by previous studies, we approached a non-label, enzyme-free and universal logic operation platform (BGG) based on G-quadruplex special structure and new nanomaterialGO in our work. Using the quenching characteristic of GO to AgNCs and the split $\mathrm{G}_{4}$-mediated fluorescence enhancement of NMM, a series of binary logic gates (OR, AND, INHIBIT, $\mathrm{XOR}$ ) were developed and verified by biological experiments. Moreover, on the basis of the same BGG platform, two nonlabel and enzyme-free combinatorial logic gates were successfully realized conceptually, including a half adder/half subtractor. More importantly, the constructed logic devices can be implemented in any enzyme-free condition without any fluorescent or quenching modification, making the logic platform more facile, economical, and cost-effective. Taken together, our design has promising application potential in many fields, such as bio-imaging and disease diagnosis, which offer new directions for future biological sciences. ${ }^{30,31}$

\section{Experimental}

\section{Materials and instrumentations}

The DNA oligonucleotides used in our experiments were purified from Sangon Biotech Co. (Shanghai, China). Their sequences are list in Table 1. Stock solutions were prepared by dissolving an appropriate amount of DNA oligonucleotides in pure water. The DNA concentration was determined by measuring the absorbance at $260 \mathrm{~nm}$. N-Methyl-mesoporphyrin IX (NMM) was purchased from J\&K Scientific Ltd. (Beijing, China) and diluted with dimethyl sulfoxide (DMSO). This prepared solution was kept away from light at $-20^{\circ} \mathrm{C}$ before use. Graphene Oxide (GO) was obtained from Nanjing XFNANO Materials Tech Co., Ltd (Nanjing, China). The others were purchased from Xi'an JingBo Bio-Technique Co. (Xi'an, China). UV-vis absorption measurements were performed on a Cary 500 Scan UV/Vis spectrophotometer (Varian, USA). The fluorescence spectra could be measured using a fluorescent scanning spectrometer for NMM at $399 \mathrm{~nm}$ excitation and $610 \mathrm{~nm}$ emission by EnSpire ELIASA from PerkinElmer USA (Shanghai, China).

\section{Basic logic gate operations}

Before operating the basic logic gates, all of the DNA solutions had been diluted $(10 \mu \mathrm{M})$ with phosphate buffer $(40 \mathrm{mM}$ phosphate, $2 \mathrm{mM} \mathrm{Mg}^{2+}, 20 \mathrm{mM} \mathrm{K}^{+}, \mathrm{pH}=7.4$ ) and separately heated at $95^{\circ} \mathrm{C}$ for $5 \mathrm{~min}$, cooled down slowly to room temperature, and stored at $4^{\circ} \mathrm{C}$. Then, for all logic devices, the platform was prepared by BGG-strand $(1 \mu \mathrm{M})$ and $\mathrm{GO}$ $(20 \mu \mathrm{g} / \mathrm{mL})$ with a buffer solution $(20 \mathrm{mM}$ Tris- $\mathrm{HCl}, 100 \mathrm{mM}$ $\mathrm{NaCl}, 10 \mathrm{mM} \mathrm{KCl}, 10 \mathrm{mM} \mathrm{MgCl} 2, \mathrm{pH}=7.5$ ) for $10 \mathrm{~min}$ at room temperature. Next, corresponding input DNA strands (1 $\mu \mathrm{M}$ IN1, IN2, IN3, IN4, IN5, IN6 and IN7) for different logic gates were mixed with the initial platform for $60 \mathrm{~min}$ at $37^{\circ} \mathrm{C}$, respectively. After that, individual final products were incubated with NMM $(1.5 \mu \mathrm{M})$ for $25 \mathrm{~min}$ at $37^{\circ} \mathrm{C}$, and the fluorescence intensity was measured in the end.

\section{Simulation of logic gates}

The sequences and the free energy of the secondary structure of each DNA strands were designed and calculated by NUPACK. ${ }^{32}$ In addition, the equilibrium concentration for each species of complex in the platform was simulated by NUPACK and Visual DSD in different logic devices systems.

\section{Results and Discussion}

Operation of basic logic gates

In our design, the basic logic gates mainly included three parts: the BGG system as the work platform, elaborate DNA strands as inputs and the fluorescence reporting signal as output. Among them, the ssDNA strand (BGG) was anchored on the $\mathrm{GO}$, working as the original platform. Especially, the BGG strand consisted of three sections ( $\mathrm{g} 1, \mathrm{c}, \mathrm{g} 2)$, in which $\mathrm{g} 1$ and $\mathrm{g} 2$ could form the G-quadruplex/NMM structure with fluorescence if the two regions were kept in close proximity. Besides, according to our previous research, very small amount of GO would not quench the fluorescence of G-quadruplex/NMM, but also enhanced it slightly, due to probe aggregation possibly caused by the adsorption of GO. However, when a functional complementary DNA strand was added as input, it could disrupt the formation of the G-quadruplex structure by DNA specific hybridization with BGG, which emitted the low fluorescent output signal of NMM. Therefore, an intelligently designed BGG strand was performed in solution with the proper concentration of the GO $(20 \mu \mathrm{g} / \mathrm{mL})$ start state, and was then involved in straightforward sequence-specific hybridization with different input strands, corresponding to its logic devices. Here, the absence and presence of DNA were specified as the inputs of " 0 " and " 1 " in the construction process, respectively. 

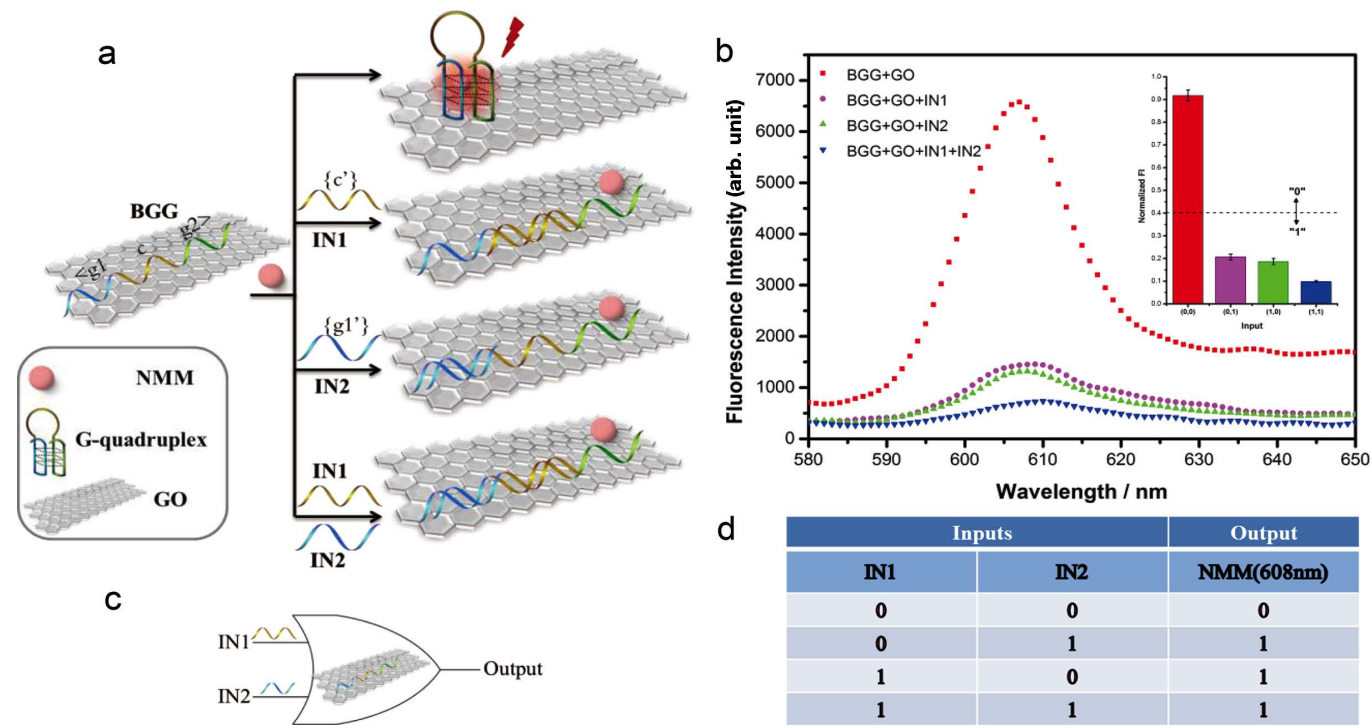

Fig. 1 "OR" logic gate (a) schematic illustration of "OR" logic gate; (b) fluorescence spectra of NMM with different combinations of inputs (insert: normalized fluorescence intensity of NMM at $608 \mathrm{~nm}$. Error bars show the standard deviation of three experiments); (c) electronic equivalent circuitry; (d) truth table of the "OR" logic gate.
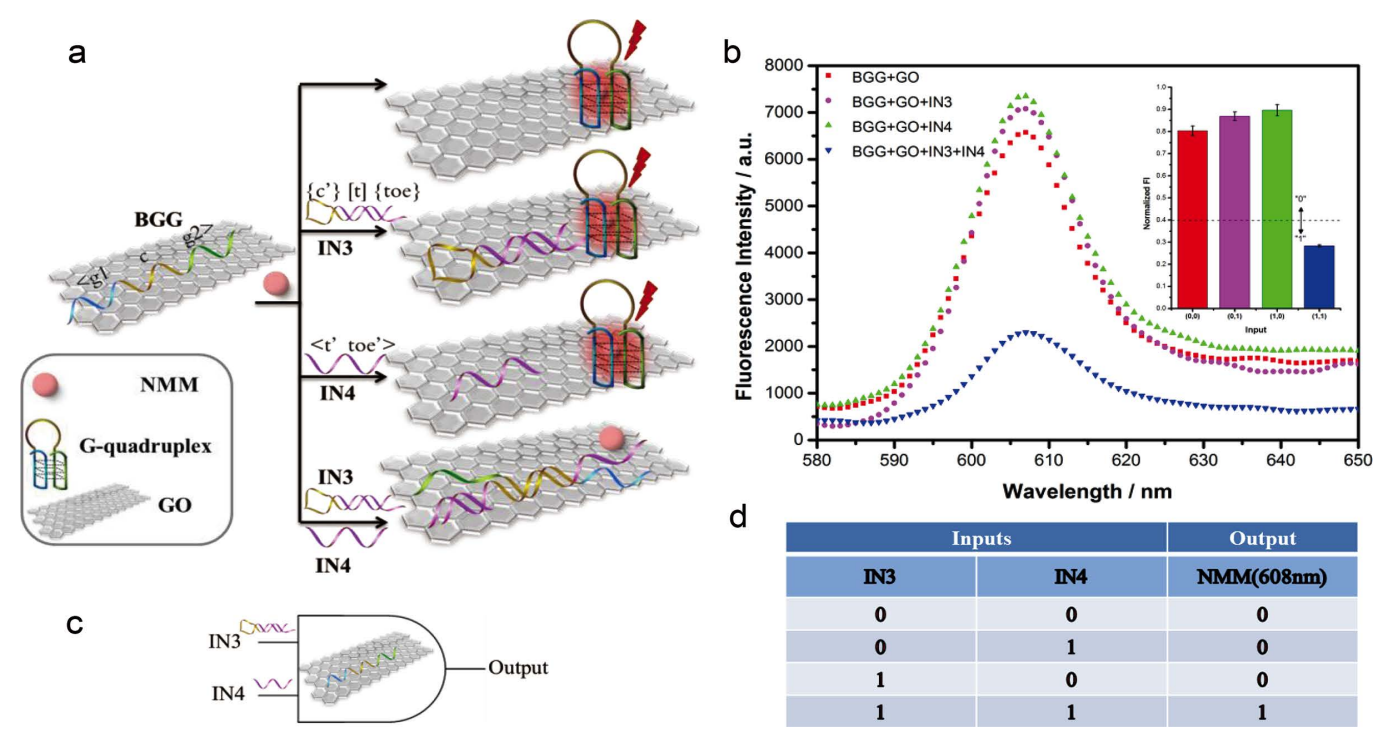

Fig. 2 "AND" logic gate (a) schematic illustration of "AND" logic gate; (b) fluorescence spectra of NMM with different combinations of inputs (insert: normalized fluorescence intensity of NMM at $608 \mathrm{~nm}$. Error bars show the standard deviation of three experiments); (c) electronic equivalent circuitry; (d) truth table of the "AND" logic gate.

Simultaneously, the fluorescence intensity of G-quadruplex/ NMM at $608 \mathrm{~nm}$, exceeding the threshold was defined as output of "0" for all the logic operation, and vice versa.

Firstly, a binary "OR" logic gate had been implemented based on the BGG platform (Fig. 1a). The two designed ssDNA strands acted as two inputs, IN1 and IN2, which could hybrid with a middle segment ( $c$, yellow region) and TTGGGTAGGG segment $\left(g 1\right.$, blue region) at the $5^{\prime}$ terminal of BGG, forming duplexes of BGG/IN1 and BGG/IN2, respectively. Herein, in the presence of IN1 or IN2, the BGG would be released from the $\mathrm{GO}$ and hardly fold into G-quadruplex due to the formation of duplex of BGG/IN1, BGG/IN2 or BGG/IN1/IN2. In this case, the fluorescence intensity (FI) of G-quadruplex/NMM had a slight increase in the optical signal (Fig. 1b, purple curve, green curve and blue curve, respectively). In contrast, there is a significantly enhanced fluorescence signal without any inputs (Fig. 1b, red curve). It was worth noting that the normalized FI at the peak above/below the threshold value of 0.40 had corresponding relations to the output " $0 " /$ " 1 ", respectively (insert of Fig. 1b). Thus, as discussed above, in the presence of any of the inputs, IN1 and IN2, a weak signal could be obtained $(Y=1)$, otherwise the FI was increased $(Y=0)$, producing the truth table (Fig. 1d), which featured the characteristics of a binary OR logic gate in Fig. 1c (Outout = IN1 + IN2).

Moreover, the experimental mechanism of the "AND" gate is demonstrate in Fig. 2a with the same working sets and different 

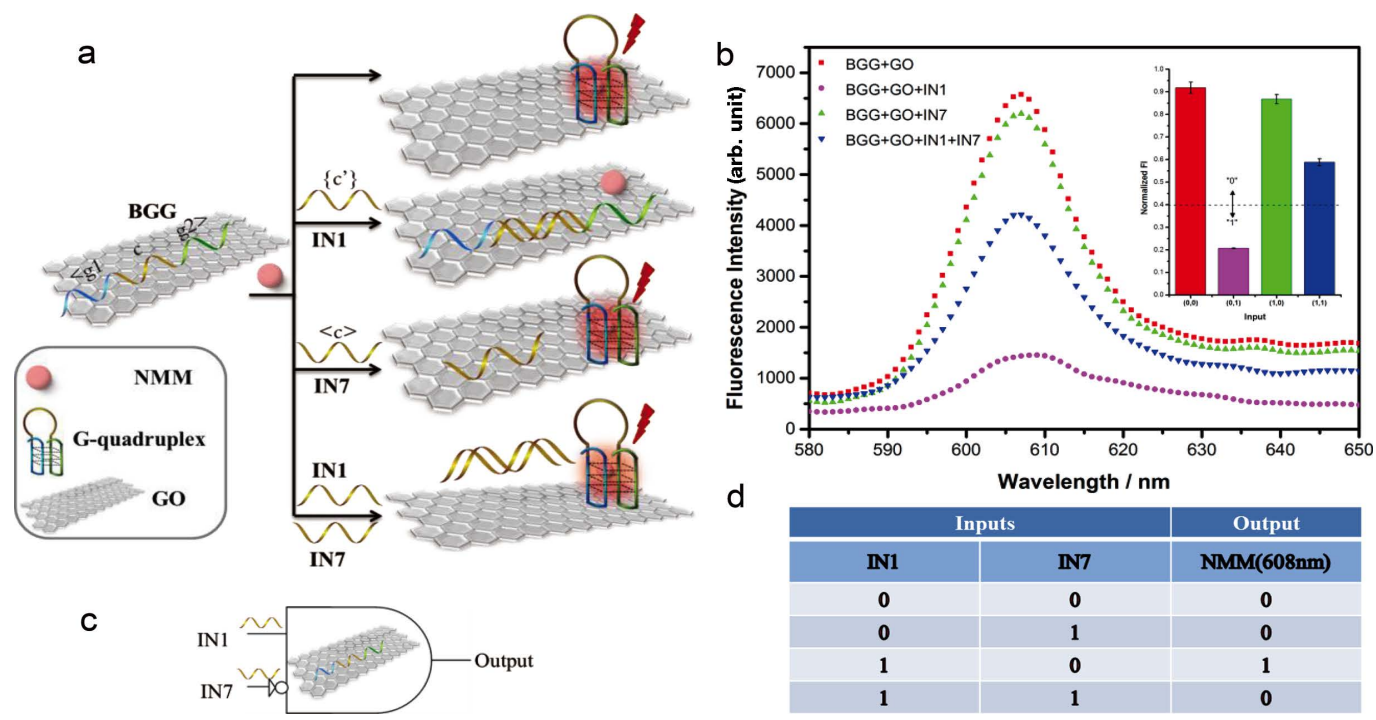

Fig. 3 "INHIBIT" logic gate (a) schematic illustration of "INHIBIT" logic gate; (b) fluorescence spectra of NMM with different combinations of inputs (insert: normalized fluorescence intensity of NMM at $608 \mathrm{~nm}$. Error bars show the standard deviation of three experiments); (c) electronic equivalent circuitry; (d) truth table of the "INHIBIT" logic gate.
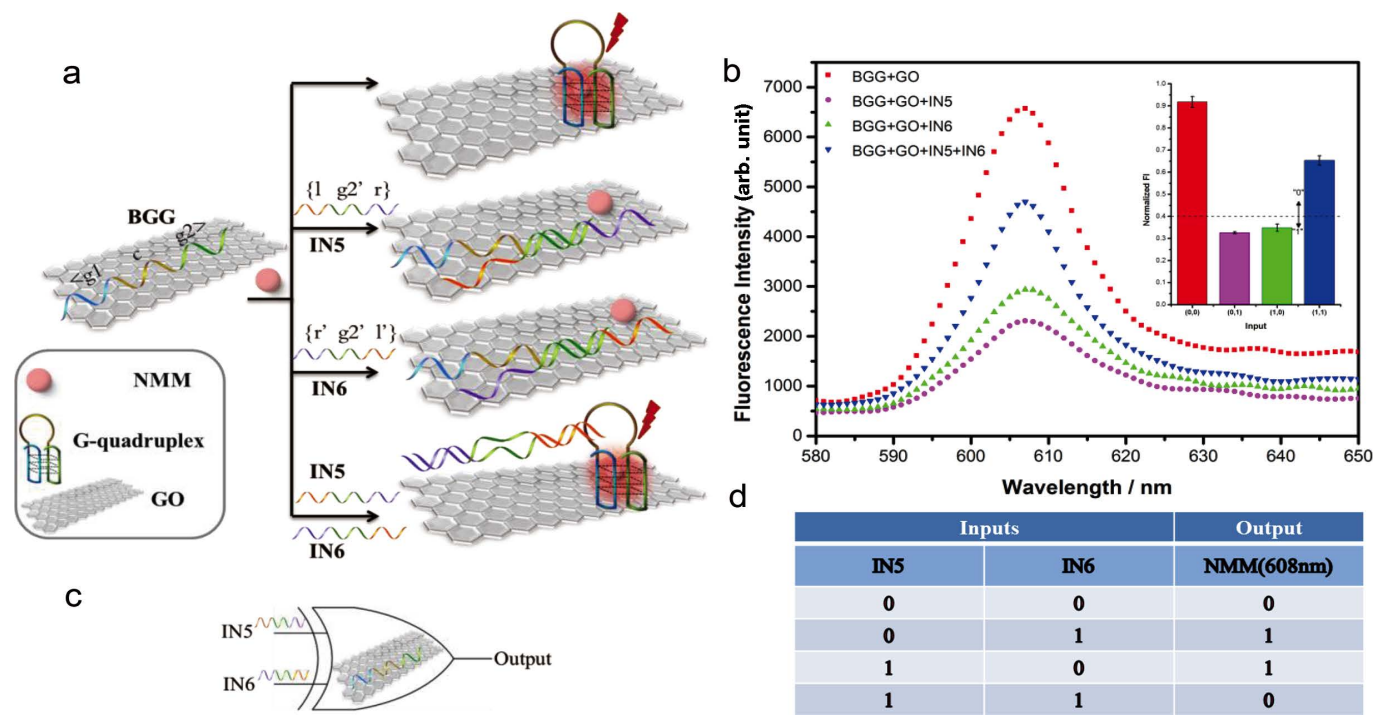

Fig. 4 "XOR" logic gate (a) schematic illustration of "XOR" logic gate; (b) fluorescence spectra of NMM with different combinations of inputs (insert: normalized fluorescence intensity of NMM at $608 \mathrm{~nm}$. Error bars show the standard deviation of three experiments); (c) electronic equivalent circuitry; (d) truth table of the "XOR" logic gate.

inputs. A hairpin structure with restricted functional regions was defined as one input, IN3, complementary to BGG in the middle ( $c^{\prime}$, yellow region). The other one, IN4, had been designed to open the stem of hairpin DNA by toehold-mediated strand displacement reaction at the $3^{\prime}$ terminal of IN3 (toe, purple region), which exposed the functional loop of IN3 $\left(c^{\prime}\right.$, yellow region). Neither of the two inputs was able to restrain the formation of G-quadruplex structure, accompanied by the high FI with G-quadruplex/NMM (Fig. 2b, red curve, purple curve and red curve, respectively), whereas the coexistence of them would hybridize with BGG in a characteristic pattern, resulting in a low optical output (Fig. 2b, blue curve). The rules of the definition of the inputs and output were similar to the above. Over all, only the input state $(1,1)$ corresponded to the true output of " $Y=1$ ", and others generated false output of " $Y=0$ " (Figs. 2c and 2d). The results were consistent with the expression of the "AND" logic gates, Output = IN3.IN4

To further prove the universality of the platform, the "INHIBIT" logic gate was also designed and illustrated by combining BGG with two inputs in Fig. 3(a). IN1 mentioned above and corresponding specific recognition sequences, IN7, had the property of priority binding ( $c$ and $c^{\prime}$, yellow region) and were defined as inputs. With respect to the inputs, IN1 also likely worked as a functional block to effectively prevent the formation of G-quadruplex/NMM (Fig. 3b, purple curve). That was, the BGG sequence would fold into a G-quadruplex/NMM 

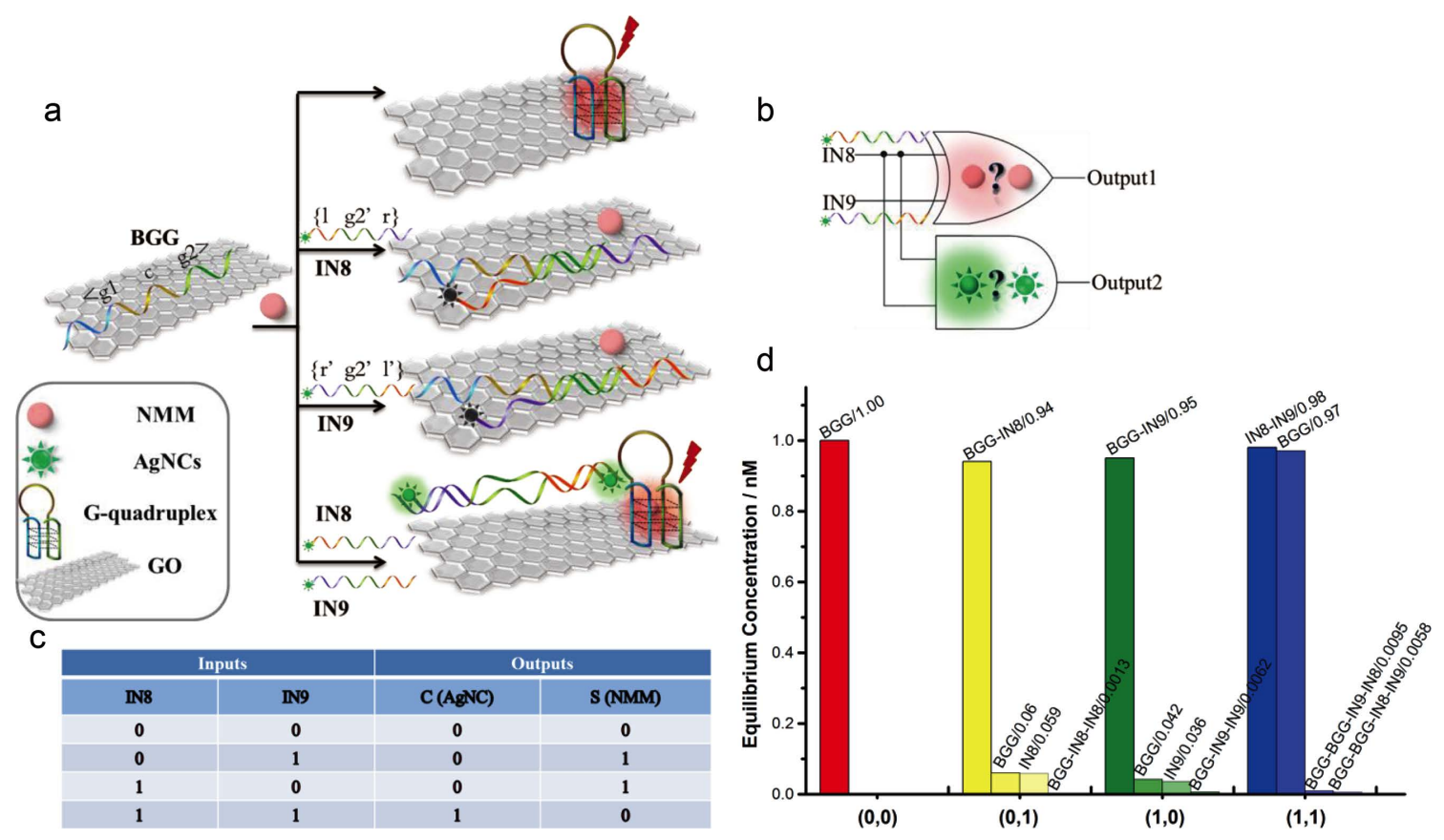

Fig. 5 "Half Adder" logic gate (a) schematic illustration of "Half Adder" logic gate; (b) electronic equivalent circuitry; (c) truth table of the "Half Adder" logic gate; (d) the simulation results of equilibrium concentration with different combinations of inputs.

in the absence of a block IN1 strand, and the fluorescence of G-quadruplex/NMM was recorded consequently (Fig. 3b, red curve, green curve and blue curve respectively). Here, the FI of test tubes containing the mixtures of IN1, IN7 (Fig. 3b, blue curve) was slightly lower than that of red curve and green curve due to weak interaction of BGG/IN1. Similarly, it had an output of " $Y=1$ " when the input was $(1,0)$, while other inputs $(0,0)$, $(0,1),(1,1)$ demonstrated output of " $Y=0$ " (Insert of Fig. 3b). In conclusion, the signal response of G-quadruplex/NMM was related to the required INHIBIT logic operation in Figs. $3 \mathrm{c}$ and 3d, Output = IN1·(IN7)'.

For the "XOR" logic gate in Fig. 4a, the sequences of inputs IN5 and IN6, were artfully designed containing complementary areas at both ends ( $l$ and $l^{\prime}$, red region; $r$ and $r^{\prime}$, purple region) and functional area in the middle ( $g 2^{\prime}$, green region). After adding any one of the inputs, IN5 or IN6, the TCGGGTTGGG segment ( $g 2$, green region) at the $3^{\prime}$ terminal of BGG would participate in hybridization reactions with a functional area of input, the formation of duplex of BGG/IN5 or BGG/IN6. In this case, the G-quadruplex could hardly come into being and then the fluorescence of NMM was weak (Fig. 4b, purple curve and green curve, respectively). However, in the coexistence of IN5 and IN6, the duplex of IN5/IN6 rather than BGG/IN5 and BGG/IN6 would prefer to form due to complementary areas, as demonstrated by the NUPACK simulation results before experiments. At the same time, the majority of free BGG turned into the G-quadruplexes configuration to emit a significantly enhanced fluorescent signal in the effect of NMM (Fig. 4b, blue curve). As shown in the insert of Fig. 4b, when the input states were $(0,0),(1,1)$, the output was " $Y=0$ ", whereas the other states $(0,1),(1,0)$ displayed the output " $Y=1$ ". In brief, in the presence of different inputs, we obtained the true table in Fig. 4d, which could meet the requirement of a "XOR" logic gate, Output $=$ IN5 $\oplus \mathrm{IN} 6=\mathrm{IN} 5 \cdot(\mathrm{IN} 6)^{\prime}+(\mathrm{IN} 5)^{\prime} \cdot \mathrm{IN} 6($ Fig. $4 \mathrm{c})$.

\section{Operation of arithmetic functions}

As important advanced logic gates, it was significant to do efficient research with a half adder/half subtractor in digital and information processing. Therefore, the arithmetic circuits in proof-of-principle studies were constructed on the same universal BGG system to implement more advanced and complex arithmetic functions. Additionally, the presence/ absence of inputs was defined as "1"/"0". The normalized FI of G-quadruplex/NMM above/below the threshold value corresponded to output "0"/" 1 ", while the signal output of AgNCs had the opposite definition. And the definition was available for half adder/half subtractor in this work below.

A half adder, which could perform an addition function on two binary digits, consisted of parallel AgNC-related XOR gate and NMM-related AND gate to code for SUM (S) and CARRY (C) digits, respectively (Fig. 5b). As shown in Fig. 5a, the half adder logic device was comprised of this BGG platform and two input strands, IN8 and IN9. They had the same nucleic acid sequences with IN5 and IN6 in "XOR" logic gate above, but stabilized with AgNCs at the $3^{\prime}$ terminal ( $l$, red region). At the initial state, BGG strand interacted with NMM, resulting in the higher fluorescence intensity. In the case of any stabilizedAgNCs input, IN8 or IN9, the hybrids with BGG was almost in a flexible single strand state $\left(g 1\right.$, blue; $c$, yellow; $l$ and $l^{\prime}$, red; $r$ and $r^{\prime}$, purple region) with very few ds-DNA regions ( $g 2$ and $g 2^{\prime}$, green region). Hence, it still anchored on the surface of $\mathrm{GO}$, leading to fluorescence quenching of AgNCs. On the other hand, the FI of G-quadruplex/NMM had an incredibly small variation due to the fact that there was not sufficient to form G-quadruplexes in the BGG/IN8 or BGG/IN9. Regarding the coexistence of IN8 and IN9, hybridization between them took place prior to their respective interactions with BGG, resulting in the separation of ds-DNA IN8/IN9 from the GO surface and a remarkable signal increase of AgNCs. Meanwhile, the two ends of BGG formed the G-quadruplex in the presence of NMM, which enhanced the fluorescent emission remarkably. 

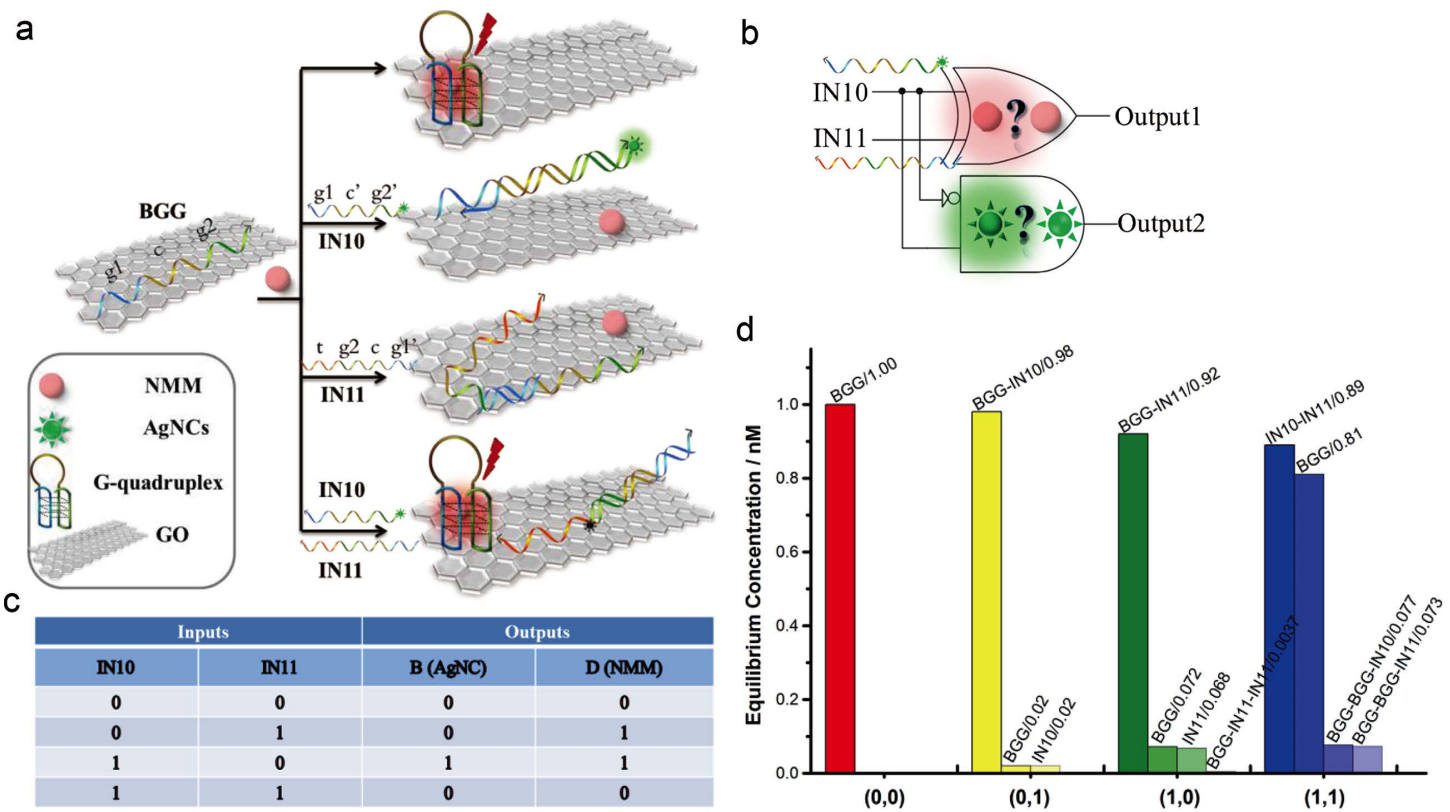

Fig. 6 "Half Subtractor" logic gate (a) schematic illustration of "Half Subtractor" logic gate; (b) electronic equivalent circuitry; (c) truth table of the "Half Subtractor" logic gate; (d) the simulation results of equilibrium concentration with different combinations of inputs.

Next, Fig. $5 \mathrm{c}$ shows the truth table with dual-output under various conditions, $(C=0, S=0)$ for input $(0,0),(C=0, S=1)$ for input $(0,1),(C=0, S=1)$ for input $(1,0)$ and $(C=1, S=0)$ for input $(1,1)$, respectively, which met the requirement of the half adder logic circuit, $C=$ IN8.IN9, $S=$ IN8 $\oplus$ IN9 $=$ IN8.(IN9) $)^{\prime}+\left(\right.$ IN8) $\cdot$ IN9'$^{\prime}$. More importantly, we could calculate the equilibrium concentration for all of the structures in the thermodynamic limit of compound system, which was crucial for designing and analyzing experiments. Here, as shown in Fig. 5d, the equilibrium concentration for each species of complex in the platform was simulated by NUPACK under different inputs. Multiple molecular structures appeared at equilibrium, and their proportion was in line with our expectations. For example, in the case of single input, the equilibrium concentration of BGG/IN8 or BGG/IN9 complex was at a higher level. But in the presence of dual inputs, the ratio of IN8 and IN9 complexes was as high as 98\%. Meantime, $97 \%$ of BGG ssDNA was in a free state, which could further interact with NMM to form G-quadruplex. The simulation results indicating that the sequences design of input and BGG platform was reasonable in our work, theoretically.

Similarly, a half subtractor could be used to implement a subtraction on two binary digits by combining a NMM-related INHIBIT gate and an AgNC-related XOR gate in parallel to produce a DIFFERENCE (D) bit and a BORROW (B) bit, respectively (Fig. 6b). Two new inputs, IN10 modified with $\mathrm{AgNCs}$ at the $5^{\prime}$ terminal ( $g 2^{\prime}$, green region) and another input IN11, were introduced on the same BGG platform in Fig. 6a. In the presence of either input, the IN10 or IN11, the split G-quadruplexe sequences of BGG could be blocked by forming the duplex of BGG/IN10 or BGG/IN11, accompanied by the low fluorescence intensity of G-quadruplexe/NMM. Note that the ds-DNA BGG/IN10 desorbs from GO and strong fluorescence emission of AgNCs was obtained when stabilizedAgNCs IN10 was added separately and hybridized with the BGG to form a duplex. Nevertheless, the coexistence of the two inputs, IN10 and IN11, could hybrid each other preferentially instead of binding to BGG, resulting in the formation of the G-quadruplex and a higher fluorescence of NMM. At the same time, the fluorescence of stabilized-AgNCs was quenched, due to the absorption function between GO and this “TTTTTTTTTTTT" longer segment at the 3 ' terminal of IN11 ( $t$, red region). To sum up, we generalized a truth table with the dual-output, $(B=0, D=0)$ for input $(0,0),(B=0, D=1)$ for input $(0,1),(B=1, D=1)$ for input $(1,0)$ and $(B=0, D=0)$ for input $(1,1)$, respectively (Fig. $6 \mathrm{c}$ ), which accorded with the feature of half subtractor, $B=\operatorname{IN} 10 \cdot(\mathrm{IN} 11)^{\prime}, D=\mathrm{IN} 10 \oplus \mathrm{IN} 11=$ IN10 $(\text { IN11 })^{\prime}+(\text { IN10 })^{\prime} \cdot$ IN11. Figure $6 \mathrm{~d}$ shows the simulation results of the sequences design similar to the previous. When input was $(0,1)$ or $(1,0)$, almost all BGG were blocked by IN10 or IN11, meaning no G-quadruplex formation in both cases. The results were consistent with our expectations, giving a further verification on the feasibility of this computing platform in theory.

\section{Conclusions}

To overcome the limits of further component-miniaturization and the operation speed of the conventional computer based on silicon-materials, biological computing is considered to be a conceptually novel idea to breakthrough to another level of information-processing technology. Combining the fluorescence intensity of G-quadruplex/NMM with the quenching ability of GO, we have successfully established a non-label, enzyme-free and universal logic platform based on DNA hybridization and molecule interaction. A series of basic logic gates have been artfully designed and verified by biological experiments, including OR, AND, INHIBIT and XOR gates. Subsequently, the library of simple gates can be considered to be the basis of more complex computational operations. Through the combination of the XOR logic gate with AND or INHIBIT gate, we have also designed a non-label, enzyme-free half adder/subtractor based on the same BGG work platform. 
Our biocomputing platform shares several distinct advantages, as follows. First of all, one characteristic of the versatile platform has good capability of DNA computing without fluorophores or quenchers, which effectively eliminates the deficiencies, including complexity, inefficiency and high-cost. Then, a library of simple gates can be implemented on the same universal platform, as well as complex operations. Therefore, the platform has better versatility for other complex logic devices by ingenious design input sequence, and certain potential applications in biocomputing and biosensor for disease diagnosis. Finally, because this new nano-material GO is facile preparation and cost effective, the proposed platform meets the requirements of future practical applications.

\section{Acknowledgements}

This research is supported by National Natural Science Foundation of China (Grant No. 61572302, No. 61272246).

\section{References}

1. L. Qian, E. Winfree, and J. Bruck, Nature, 2011, 475, 368.

2. P. Ball, "Chemistry Meets Computing", 2000, 406, 118.

3. D. S. A Prasanna and U. Seiichi, Nat. Nanotech., 2007, 2, 399.

4. A. S. Sadek, K. Nikolić, and M. Forshaw, Nanot, 2004, 15, 192.

5. Z. Hu, J. Jian, Y. Hua, D. Yang, Y. Gao, J. You, Z. Wang, Y. Chang, K. Yuan, and Z. Bao, Sens. Actuators, B, 2018, 273, 559.

6. S. Xu, H. Li, Y. Miao, Y. Liu, and E. Wang, Npg. Asia. Mater, 2013, 5, e76.

7. H. Li, Y. Liu, S. Dong, and E. Wang, Npg. Asia. Mater., 2015, 7, 1009.

8. R. Orbach, F. Wang, O. Lioubashevski, R. D. Levine, F. Remacle, and I. Willner, Chem. Sci., 2014, 5, 3381.

9. D. Fan, J. Zhu, Y. Liu, E. Wang, and S. Dong, Nanoscale, 2016, $8,3834$.

10. S. Zhang, K. Wang, C. Huang, Z. Li, T. Sun, and D. M. Han, Nanoscale, 2016, 8, 15681.

11. J. Zhu, L. Zhang, T. Li, S. Dong, and E. Wang, Adv. Mater.,
2013, 25, 2440.

12. M. Wang, Z. Mao, T. S. Kang, C. Y. Wong, J. L. Mergny, C. H. Leung, and D. L. Ma, Chem. Sci., 2016, 7, 2516.

13. S. Lin, W. Gao, Z. Tian, C. Yang, L. Lu, J. L. Mergny, C. H. Leung, and D. L. Ma, Chem. Sci., 2015, 6, 4284.

14. C. H. Lu, A. Cecconello, and I. Willner, J. Am. Chem. Soc., 2016, 138, 5172.

15. Z. G. Wang, J. Elbaz, F. Remacle, R. D. Levine, and I. Willner, Proc. Natl. Acad. Sci. U. S. A., 2010, 107, 21996.

16. H. Pei, L. Liang, G. Yao, J. Li, Q. Huang, and C. Fan, Angew. Chem. Int. Edit., 2012, 51, 9020.

17. S. Zhang, K. Wang, C. Huang, and T. Sun, Nanoscale, 2015, 7, 20749.

18. X. Zhang, B. Ding, H. Wu, J. Wang, and H. Yang, Anal. Sci., 2017, 33, 165.

19. B. Ding, C. Liu, Q. Wu, Y. Wang, L. Li, and H. Yang, Anal. Sci., 2018, 34, 259.

20. Y. Zhang, L. Wang, Y. Wang, and Y. Dong, Sensors, 2018, 18, 2179.

21. J. Li, Y. Jia, J. Zheng, W. Zhong, G. Shen, R. Yang, and W. Tan, Chem. Commun., 2013, 49, 6137.

22. D. Bai, D. Ji, J. Shang, Y. Hu, J. Gao, Z. Lin, J. Ge, and Z. Li, Sens. Actuators, B, 2017, 252, 1146.

23. L. Wang, Y. Wen, L. Li, X. Yang, N. Jia, W. Li, J. Meng, M. Duan, X. Sun, and G. Liu, Biosens. Bioelectron., 2018, 115, 91.

24. X. F. Zhang, H. M. Xu, L. Han, N. B. Li, and H. Q. Luo, Anal. Sci., 2018, 34, 149.

25. D. L. Ma, L. Lu, S. Lin, B. He, and C. H. Leung, J. Mater. Chem. B, 2014, 3, 348.

26. D. M. Kolpashchikov, J. Am. Chem. Soc., 2008, 130, 2934.

27. L. Zhou, H. Cheng, J. E. Wang, and R. J. Pei, Chin. J. Anal. Chem., 2016, 44, 13.

28. X. Liu, F. Wang, R. Aizen, O. Yehezkeli, and I. Willner, J. Am. Chem. Soc., 2013, 135, 11832.

29. Q. Mei, C. Jiang, G. Guan, K. Zhang, B. Liu, R. Liu, and Z. Zhang, Chem. Commun., 2012, 48, 7468.

30. T. Konry and D. R. Walt, J. Am. Chem. Soc., 2009, 131, 13232.

31. E. Katz, J. Wang, M. Privman, and J. Halámek, Anal. Chem., 2012, 84, 5463.

32. R. M. Dirks, J. S. Bois, J. M. Schaeffer, E. Winfree, and N. A. Pierce, Siam Rev., 2007, 49, 65. 